\title{
Maximization of Measurement Sensitivity and Reading Range of Passive RF Sensors from Complex Impedance Optimization
}

\author{
Victor ENGELHARDT ${ }^{* \dagger}$, Camille JOUVAUD ${ }^{* \dagger}$, François SARRAZIN ${ }^{* \dagger}$, \\ Christophe DELAVEAUD ${ }^{* \dagger}$ and Hervé AUBERT ${ }^{\ddagger}$ \\ ${ }^{*}$ CEA-LETI, Grenoble, France \\ ${ }^{\dagger}$ University of Grenoble-Alpes, Grenoble, France \\ ${ }^{\ddagger}$ LAAS-CNRS, University of Toulouse, CNRS, INPT, Toulouse France \\ Email: victor.engelhardt@cea.fr
}

\begin{abstract}
This paper reports a method to improve the performances of passive Radiofrequency sensors in terms of reading range and measurement sensitivity. More specifically the impedance profile of the sensing device that maximizes the measurement sensitivity and reading range is derived. This profile allows predicting the largest achievable reading range and the highest measurement sensitivity that can be achieved by a given sensor antenna. The proposed original method is illustrated through the design of a wireless and passive temperature sensor. Experimental results are reported for validation purposes.
\end{abstract}

\section{INTRODUCTION}

The Internet of Things is a promising research area for the next years. 50 billions of connected objects are expected in 2020. Among them, wireless sensors are required for applications like body and environmental monitoring. The variety of applications leads to a huge panel of technical constraints. In this context, chipless and passive (i.e., batteryless) Radiofrequency sensors present numerous advantages, such as, unlimited energy autonomy, long-term measurement stability and low cost of fabrication. The wireless interrogation of such sensors can be performed from the measurement of radar signature. As illustrated in Fig. 1, the technique consists in transmitting an electromagnetic wave in the direction of the sensor and deriving the measurand, i.e., the physical or chemical quantity of interest, from the signal backscattered by the Radiofrequency sensor (see, e.g., [1]). It allows the simultaneous wireless interrogation of many sensors, the realtime monitoring of each sensor and provides a simple sensor identification technique. However the well-known limitations of such sensors are the short reading range (typically of few tens meters) and the low measurement sensitivity with respect to the measurand. In this communication, a novel approach is reported for maximizing both the reading range and the measurement sensitivity of passive Radiofrequency sensors.

\section{Reading Range Maximization of Passive RADIOFREQUENCY SENSORS}

The Radiofrequency (RF) power $P_{r}$ received by the reader from the electromagnetic backscattering of a target (here a

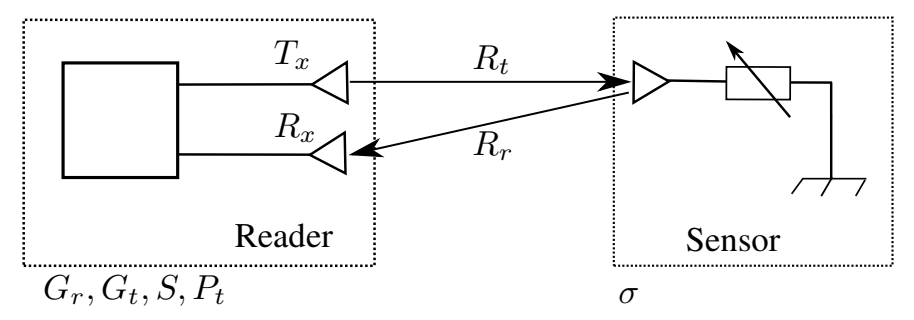

Fig. 1. Wireless Interrogation Setup of RF Passive Sensors

passive RF sensor assumed to be polarization matched) is given by [2]:

$$
P_{r}=\sigma \frac{G_{t} G_{r} \lambda^{2}}{(4 \pi)^{3} R_{t}^{2} R_{r}^{2}} P_{t},
$$

where $\sigma$ is the Radar Cross Section (RCS) of the target, $G_{t}$ and $G_{r}$ are the gains of the radar's transmitting $\left(T_{x}\right)$ and receiving ( $R_{x}$ ) antennas, respectively, $R_{t}$ is the distance between the $T_{x}$ antenna and the sensor, $R_{r}$ is the distance between the sensor and the $R_{x}$ antenna, $P_{t}$ denotes the power transmitted by the reader and $\lambda$ is the free space wavelength at the operating frequency. If the transmission and reception share the same antenna $\left(G_{t}=G_{r}=G\right)$, the reading range $R=R_{t}=R_{r}$ is then derived as follows :

$$
R=\sqrt[4]{\sigma \frac{G^{2} \lambda^{2} P_{t}}{(4 \pi)^{3} S}}
$$

where $S$ is the receiver sensitivity, that is, the minimum power required by the radar reader for processing a specified received signal having a specified signal-to-noise ratio. The passive RF sensor shown in Fig. 1 is typically composed of an antenna and a sensing device, that is, a load impedance which changes when the measurand varies. The RF electromagnetic field backscattered by the sensor antenna can be decomposed into two scattering modes: (1) the so-called structural mode $A_{s}$ which does not depend on the sensing device impedance and, (2) the antenna mode which is related to the impedance 
mismatch at the input port of the antenna. The RCS of the RF sensor can be written as follows [3]:

$$
\sigma=\frac{\lambda^{2} G_{a}^{2}}{4 \pi}\left|\Gamma-A_{s}\right|^{2}
$$

with $\Gamma=\left(Z-Z_{a}^{*}\right) /\left(Z+Z_{a}\right)$ where $Z_{a}$ and $G_{a}$ denote the impedance and the gain of the sensor antenna while $Z$ is the sensing device impedance. According to Eq. 2, the reading range is a function of $\sigma$ and consequently, there are two possibilities for enhancing this range: (1) to increase the gain $G_{a}$ and/or (2) to maximize the factor $\left|\Gamma-A_{s}\right|^{2}$ from the appropriate choice of the sensing device impedance. The latter possibility requires the knowledge of the key parameter $A_{s}$. Several works have been reported on the derivation of this parameter for simple geometrical structures (see, e.g., [4]). For complex antenna geometry, a graphical approach exists [5] and consists in simulating the antenna loaded by three different impedances. Once $A_{s}=a_{s} \exp \left(i \theta_{A_{s}}\right)$ is found, the complex impedance $Z_{\text {min }}$ such as $\Gamma\left(Z_{\text {min }}\right)=A_{s}$ derives as follows :

$$
Z_{\text {min }}=\frac{Z_{a}^{*}+Z_{a} A_{s}}{1-A_{s}}
$$

According to Eq. 3, when the antenna is loaded by this complex impedance $Z_{\text {min }}$, the electromagnetic backscattering from the RF sensor is theoretically canceled. Moreover, from Eq. 3 the impedance $Z_{\max }$ that maximizes the RCS (and the reading range) can also be derived as follows :

$$
Z_{\text {max }}=\frac{Z_{a}^{*}+Z_{a} \exp \left(i\left(\theta_{A_{s}}+\pi\right)\right)}{1-\exp \left(i\left(\theta_{A_{s}}+\pi\right)\right)}
$$

\section{Maximization of Passive Sensor Sensitivity}

The objective is here to determine how the complex impedance of the sensing device must vary in response to a measurand variation in order to maximize the measurement sensitivity. The first stage $(n=0)$ consists of computing the Radar Cross Section when the antenna is loaded by the impedance $Z_{0}=Z_{\max }$ given by Eq. 5; In the second stage $(n=1)$, the impedance $Z_{0}$ is replaced by the impedance $Z_{1}=Z_{0}+\Delta Z$ with $\Delta Z=\epsilon e^{i \theta_{1}}$ where $\epsilon<<\left\|Z_{\max }-Z_{\text {min }}\right\|$ is fixed and $\theta_{1}$ is determined such that the Radar Cross Section difference $\sigma\left(Z_{0}\right)-\sigma\left(Z_{1}\right)$ is maximal; The third stage $(n=2)$ consists in replacing $Z_{1}$ by $Z_{2}=Z_{1}+\Delta Z$ with $\Delta Z=\epsilon e^{i \theta_{2}}$ where $\theta_{2}$ is chosen such that $\sigma\left(Z_{1}\right)-\sigma\left(Z_{2}\right)$ is maximal, and so on... The process is stopped at the stage $N(n=N-1)$ when $\sigma\left(Z_{N}\right)$ is minimal. For any $n \in[0, N]$ the measurement sensitivity $\left|\sigma\left(Z_{n}\right)-\sigma\left(Z_{n-1}\right)\right| / \epsilon$ is maximized. Consequently, as the measurand varies, the complex impedance of the sensing device must take values in the set $\left\{Z_{0}, \ldots, Z_{N}\right\}$ for achieving the highest measurement sensitivity with respect to the measurand.

\section{Application to the Design of RF Temperature SENSORS}

For illustration purposes we present now the design of a wireless and passive temperature Radiofrequency $(869.8 \mathrm{MHz})$ sensor by applying the method described in Sections II and

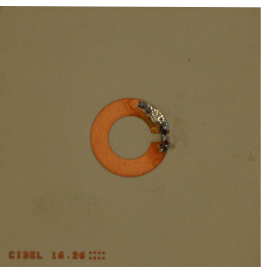

(a) Top view

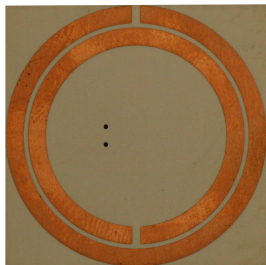

(b) Bottom view
Fig. 2. Photography of the antenna used for the RF temperature sensor application

TABLE I

Complex IMPEDANCE of THE LOADING SENSING DeVICE WHICH Minimizes $\left(Z_{\min }\right)$ AND MAXIMIZES $\left(Z_{\max }\right)$ THE RCS OF THE SENSOR ANTENNA AT $869.8 \mathrm{MHZ}$

\begin{tabular}{|c||c|}
\hline Impedance of the loading sensing device $[\Omega]$ & RCS of the sensor $\left[\mathrm{mm}^{2}\right]$ \\
\hline \hline$Z_{\min }=0.26-66 i$ & 82.65 \\
\hline$Z_{\max }=41.25 i$ & $2.42 .10^{4}$ \\
\hline
\end{tabular}

III. The chosen antenna, shown in Fig. 2, is composed of a planar loop printed at one side of a ROGERS 3003 Substrate of dimension $30 \times 30 \times 0.75 \mathrm{~mm}^{3}$. A Split Ring Resonator (SRR) is printed at the other side of the substrate. The input impedance $Z_{a}$ at $868 \mathrm{MHz}$ of this antenna is found to be of $53.9 \Omega+i 3.4 \Omega$. Using the method proposed in Section II, the two impedances $Z_{\min }$ and $Z_{\max }$ that respectively minimize and maximize the RCS of the sensor are first computed. The obtained values are reported in Table I, as well as the corresponding RCSs. The complex impedance $Z_{\text {opt }}$ of the sensing device that allows achieving the highest measurement sensitivity with respect to the measurand is derived from the method described in Section III. The real $\left(R_{o p t}\right)$ and imaginary $\left(X_{o p t}\right)$ parts of this complex impedance are presented in Fig. 3. These results are very useful to select the sensing device which is the most appropriate to the chosen antenna for maximizing the measurement sensitivity. As the measurand varies, the resistance of the sensing device must ideally range from $0 \Omega$ to $50 \Omega$ while its reactance must vary between $40 \Omega$ and $-60 \Omega$.

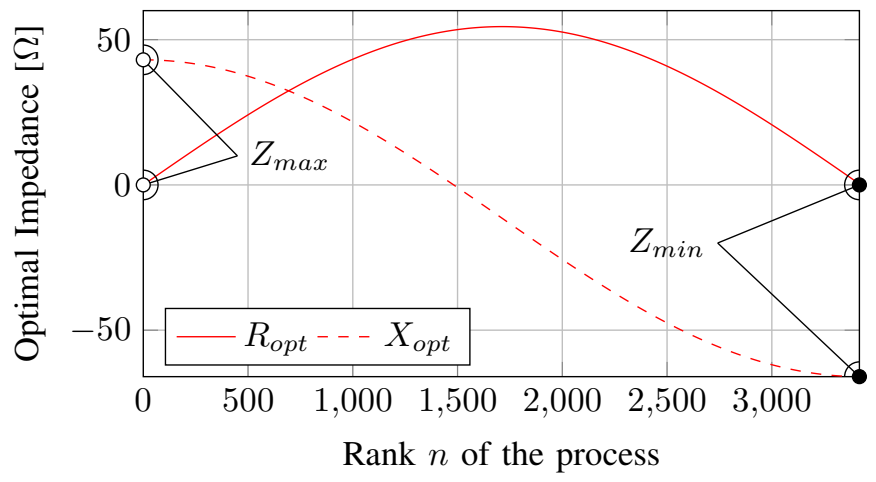

Fig. 3. Real (solid curve) and imaginary (dashed curve) parts of the impedance $Z_{\text {opt }}$ found by the process presented Section III for maximizing the measurement sensitivity 


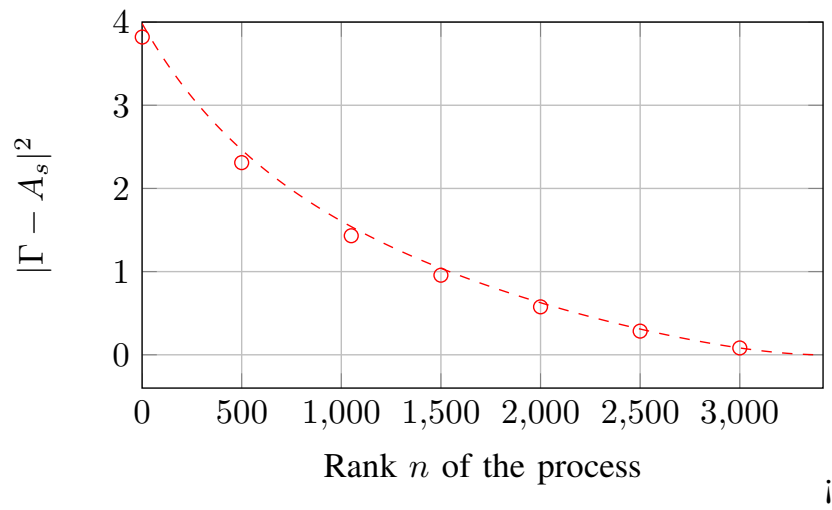

Fig. 4. $\left|\Gamma-A_{s}\right|^{2}$ versus the rank $n$ of the process described Section III (dashed line). Circles indicate the values obtained from the full-wave electromagnetic simulation (CST) of the chosen antenna loaded by the complex impedance $Z_{\text {opt }}$ at rank $\mathrm{n}=1,500,1000,1500,2000,2500$ and 3000

Next the factor $\left|\Gamma-A_{s}\right|^{2}$ is computed for the complex impedance $Z_{\text {opt }}$. The results are shown in Fig. 4 and are found in very good agreement with results obtained from the fullwave electromagnetic simulation (CST Microwave Studio) of the chosen antenna loaded by the complex impedance $Z_{o p t}$. In order to reach the highest measurement sensitivity when the temperature varies, we must ideally select a sensing device having the impedance $Z_{o p t}$. A resistance variation close to the required one is achieved by the high frequency HFT1220-12R0 thermistor. The resistance of this sensing device varies from $3 \Omega$ to $61.5 \Omega$ when the temperature varies between $-40{ }^{\circ} \mathrm{C}$ and $80{ }^{\circ} \mathrm{C}$. The reactance of this thermistor does not range from $40 \Omega$ to $-60 \Omega$, as required. We shall not report in this paper the design of the required reactance. However adding a temperature-independent reactance of $40 \Omega$ in series with the thermistor is sufficient for enhancing the reading range and measurement sensitivity. As a matter of fact, it can be observed from Fig. 5 that the RCS of the antenna loaded by the thermistor in series with this reactance is higher than the RCS of the antenna loaded by the thermistor alone. Moreover the measurement sensitivity is significantly improved when the reactance is present $\left(\Delta \sigma / \Delta T\right.$ is of $226 \mathrm{~mm}^{2} /{ }^{\circ} \mathrm{C}$ with the reactance and of $114 \mathrm{~mm}^{2} /{ }^{\circ} \mathrm{C}$ without reactance). Sections II and III were applied to the chosen antenna and the corresponding $Z_{\min }$ and $Z_{\max }$ were calculated. For experimentally illustrating the advantageous impact of a reactance in series with the resistive thermistor, the RCS of the antenna loaded by an inductor of $10 \mathrm{nH}$ in series with various resistances were measured. The measurement was performed in an anechoic chamber. A Vector Network Analyzer with two calibrated antennas were used as a reader. The RF sensor was placed at 4 meters in front of the reader. Measurement results are displayed in Fig. 6. The largest achievable RCS of the sensor is found to be of $24000 \mathrm{~mm}^{2}$ as predicted from simulation. Moreover it can be derived from this result and Eq. 2 that the reading range is increased by $25 \%$ when the reactance is present.

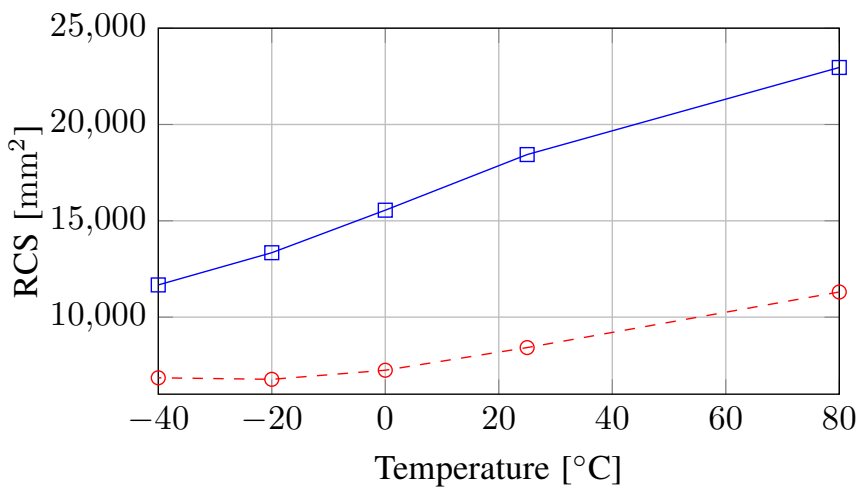

Fig. 5. Computed RCSs of the antenna at $868 \mathrm{MHz}$ loaded by the thermistor in series with this reactance (blue curve) and of the antenna loaded by the thermistor only (red curve)

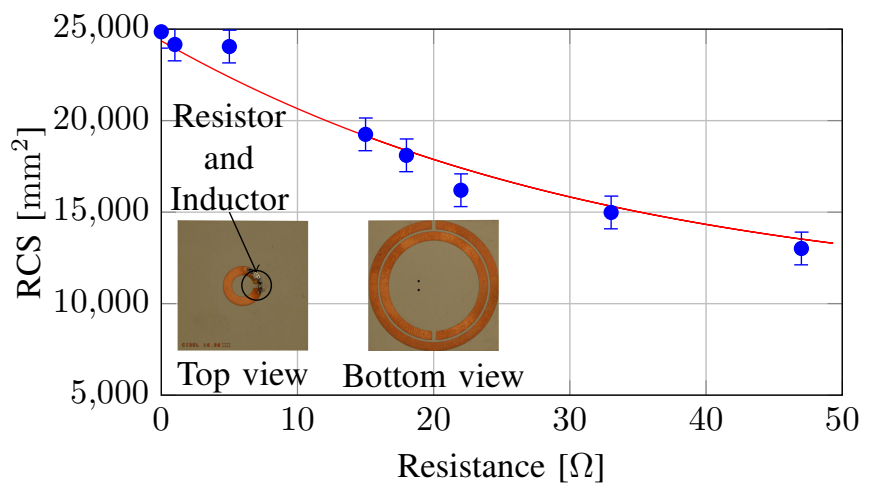

Fig. 6. Measured RCSs at $868 \mathrm{MHz}$ of the antenna loaded by a resistance in series with an inductor of $10 \mathrm{nH}$ as a function of the resistance value. Measurement results are in blue while simulation results are in red

\section{CONCLUSION}

In this paper, a new method was introduced to maximize both the reading range and the measurement sensitivity of wireless and passive Radiofrequency sensors. An application to the design of a temperature sensor was reported. Simulation and measurement results were in good agreement. It was shown that an appropriate choice of sensing device reactance allows improving simultaneously the wireless measurement sensitivity and reading range. Moreover the proposed method allows selecting the optimal sensing device for a given sensors antenna.

\section{REFERENCES}

[1] S. Bouaziz, F. Chebila, A. Traille, P. Pons, H. Aubert, and M. M. Tentzeris, "Novel Microfluidic Structures for Wireless Passive Temperature Telemetry Medical Systems Using Radar Interrogation Techniques in Ka-Band," IEEE Antennas and Wireless Propagation Letters, vol. 11, pp. 1706-1709, 2012.

[2] C. Balanis, Modern Antenna Handbook. Wiley.

[3] R. Green, The General Theory of Antenna Scattering, Columbus, 1963.

[4] S. Dike and D. King, "The Absorption Gain and Back-Scattering Cross Section of the Cylindrical Antenna," Proceedings of the IRE, vol. 40, no. 7, pp. 853-860, Jul. 1952.

[5] C. C. Yen, A. E. Gutierrez, D. Veeramani, and D. v. d. Weide, "Radar Cross-Section Analysis of Backscattering RFID Tags," IEEE Antennas and Wireless Propagation Letters, vol. 6, pp. 279-281, 2007. 